\title{
Study of anemic pattern in women of reproductive age group and elderly persons in and around Mahabubnagar, Telangana State
}

\author{
Mohammed Abdul Wahab ${ }^{1}$, Yogender Patwari,"* \\ ${ }^{1}$ Assistant Professor, Apollo Institute of Medical Sciences and Research, Hyderabad, Telangana, ${ }^{2}$ Assistant Professor, Dept. of \\ Pathology, Mandya Institute of Medical Sciences, Mandya, Karnataka, India
}

*Corresponding Author:

Email: yogi5300@gmail.com

\begin{abstract}
Introduction: Anemia leads to loss in physical function, affecting quality of life and has substantial social and economic effects. Anemia is a sign, not a diagnosis, an evaluation is almost always warranted to identify the underlying cause.

Objectives: Anemia in women of reproductive group can lead to maternal and child mortality and in elderly there is greater risk of co-morbidities, impaired functional status and greater risk of death. The objective of this study is to calculate the prevalence of anemia and pattern of anemia in this age groups.

Materials and Methods: A prospective study was undertaken at SVS Medical College and Hospital Mahabubnagar. Women of reproductive age group (16-44 years) and elderly (>60 years) were studied. Laboratory investigations like hemoglobin estimation by Cyanmethhemoglobin method, hematocrit (packed cell volume) and peripheral smear examination was done along with other tests as felt necessary to fix the pattern.

Results: Microcytic hypochromic anemia accounting for $81 \%$ in women of reproductive age group and $44 \%$ in elderly followed by normocytic normochromic anemia accounting for $10 \%$ and $19 \%$ respectively.

Iron deficiency anemia accounted for $69 \%$ in women of reproductive age group and $48 \%$ in elderly followed by anemia of chronic disease accounting for $12 \%$ and $38 \%$ respectively.

Conclusion: Confirming the type of anemia is critical to direct the investigation for profiling the etiology since it is well known that the treatment of anemia goes a long way in improving the overall outcome and quality of life.
\end{abstract}

Keywords: Anemia, Women of reproductive age group, Elderly, Serum ferritin.

\section{Introduction}

Anemia is a global public health problem affecting both developing and developed countries with major consequences for human health as well as social and economic development. It occurs in all age groups, but is more prevalent in pregnant women and young children. In 2002, iron deficiency anemia (IDA) was considered to be among the most important contributing factors to the global burden of disease. ${ }^{1}$

Anemia is an indicator of both poor nutrition and poor health. The most dramatic health effects of anaemia, i.e., increased risk of maternal and child mortality due to severe anemia, have been well documented. . $^{2-4}$

$\mathrm{Hb}$ concentration is the most reliable indicator of anemia at the population level. Measuring $\mathrm{Hb}$ concentration is relatively easy and inexpensive. However, anemia can be caused by factors other than iron deficiency.

In settings where iron deficiency is not the only cause of anaemia, approaches that combine iron interventions with other measures are needed. Strategies should include addressing other causes of anemia, 5,6 and should be built into the primary health care system and existing programmes.

\section{Objectives}

1. To calculate the prevalence of anemia in women of reproductive age group and elderly patients in and around Mahabubnagar, Telangana state.
2. To study the pattern of anemia in these patients.

Previous Studies by Health Workers: In 1967 study was done by McFarlene DB et al in West of Scotland about the incidence of iron deficiency with and without anemia in woman in general practice. They found that incidence of iron deficiency anemia in 500 women in an urban general practice was found to be 8.2 percent. $^{7}$

In 1971 JD Cook, J Alvarado study on nutritional deficiency and anemia in Latin America. A collaborative study on nutritional anemia in third trimester pregnancy was performed in seven Latin American countries. Iron deficiency was found in $48 \%$ of pregnant women, and vitamin B12 deficiency anemia was found in $15.1 \%$. Anemia as defined by current WHO criteria, was found in $38.5 \%$ of pregnant women, $17.3 \%$ of non pregnant women and $3.9 \%$ of $\operatorname{men}^{8}$

Celestin Roux et al in 1987 reported particularly high prevalences for men in all age groups, ranging from $42.8 \%$ in men aged between 70 and 74 years to $59.5 \%$ in men 85 years. ${ }^{9}$

Fleming, 1989, Brabin et al., 1990 stated that anaemia during pregnancy is associated with an increased risk of intrauterine growth retardation, premature delivery and low birth weight, resulting in an increase in perinatal mortality. ${ }^{10}$

Elis et al in 1996 have shown that most common anemia in elderly is normocytic normochromic type ${ }^{11}$

Katai et al in 1996 studied and stated that iron deficiency is common in women of reproductive age 
even in developed countries. ${ }^{12}$

Jack M. Guralnik et al in 2004 revealed that overall, $11.0 \%$ of men and $10.2 \%$ of women 65 years and older and living in the community are anaemic according to WHO criteria. ${ }^{13}$

Artz AS et al in 2004 showed that $14 \%$ to $50 \%$ of anemic elderly had no obvious underlying cause. ${ }^{14}$

World health organization criteria for anemia are haemoglobin less than $12 \mathrm{~g} / \mathrm{dl}$ in women and less than $13 \mathrm{~g} / \mathrm{dl}$ in men. ${ }^{15}$

Screening Tests: Blood counts, peripheral smear examination, PCV (Packed cell volume), MCV (Mean corpuscular volume), $\mathrm{MCH}$ (Mean corpuscular hemoglobin) and MCHC (Mean corpuscular hemoglobin concentration) are screening tests.

Other Diagnostic Tests: Serum ferritin, vitamin B12 assay, folic acid assay and bone marrow examination.

\section{Materials and Methods}

Source of Data: All the patients for whom investigations were sent to the central lab in SVS Medical College and Hospital from September 2010 to August 2012.

Method of Collection of Data: A prospective study of 150 patients, 100 cases in women of reproductive age group (16-50 years) and 50 cases in elderly people (>60) satisfying the inclusion criteria, managed by Departments of SVS Medical College Hospital were studied.

A detailed history of included patients was elicited. A complete general physical examination and systemic review of the patient was undertaken.

The following investigations were carried out for all the patients.

1. Haemoglobin estimation and Packed cell volume (PCV)

2. Mean corpuscular volume (MCV), mean corpuscular hemoglobin concentration (MCHC), mean corpuscular hemoglobin (MCH).

3. Peripheral smear for blood picture

4. Serum ferritin

5. Bone marrow studies (aspiration/biopsy) was carried out in patients with Blood Smear showing immature white cells or nucleated red cells or Indeterminate status of iron stores unexplained progressive or unresponsive anemia.

6. Vitamin B12 and folate assays were done in higher centres for dimorphic anemia and macrocytic anemia or in patients with normocytic or microcytic blood picture in whom no other cause could be found.

\section{Inclusion Criteria}

1. Women of reproductive age group (16-44 years)

2. Elderly ( $>60$ years both male and female)

3. $\mathrm{Hb}<13 \mathrm{gm} / \mathrm{dl}$ in males, $\mathrm{Hb}<11 \mathrm{gm} / \mathrm{dl}$ in pregnant females, $\mathrm{Hb}<12 \mathrm{gm} / \mathrm{dl}$ in non pregnant women)

\section{Exclusion Criteria}

1. Patients with a history of recent transfusion.

2. Patients who have undergone major surgical procedure in the past 3 months

3. Patients who were on haematinics

Study Design: Prospective study

Sample Size: 3508 subjects for whom investigations were done at central lab from September 2010 at SVS Medical College Hospital were selected for the study of prevalence.

150 patients were selected for the study of pattern of anemia as per inclusion and exclusion criteria adopted for this study.

Out of 2372 subjects taken in women of reproductive age group for studying the prevalence of anemia 78\% (1873 subjects) were non pregnant and $22 \%$ (529 subjects) were pregnant.

Out of 1136 subjects taken in the elderly for studying the prevalence of anemia 56\% (637 subjects) were elderly males and $44 \%$ (499 subjects) were elderly females.

Pregnant women had a prevalence of $72 \%$ where as non pregnant women had a prevalence of $70 \%$.

It has been noted that prevalence of anemia was higher in elderly females accounting for $81 \%$ (517 out of 637 subjects) followed by elderly males accounting for $71 \%$ (356 out of 499 subjects). Out of 1136 subjects 873 were anemic.

Out of 100 cases taken for studying the pattern of anemia in women of reproductive age group 67 were non pregnant and 33 of them were pregnant women.

It has been noted that microcytic hypochromic anemia (81\%) was the most common type of anemia in women of reproductive age group followed by normocytic normochromic anemia (12\%). Macrocytic anemia (4\%) was the third commonest type of anemia in this age group. Dimorphic anemic (3\%) was last.

Microcytic hypochromic anemia $(88 \%)$ was the most common pattern of anemia based upon the peripheral smear followed by dimorphic $(6 \%)$ and macrocytic anemia (6\%) in 33 subjects of pregnant women.

Iron deficiency was found to be the most common cause of anemia followed by anemia due to chronic diseases.

Bone marrow was done in 4 cases with peripheral smear features of microcytic and macrocytic anemia Iron deficiency anemia $(88 \%)$ was found to be the most common cause of anemia in pregnant women followed by B12 deficiency and B12 + iron deficiency anemia both accounting for $6 \%$ each.

Out of 50 subjects taken in the elderly to study the pattern of anemia 30 subjects were females and 20 subjects were males.

It has been noted in the present study that microcytic hypochromic anemia (44\%) is the most common type of anemia in elderly closely followed by normocytic hypochromic anemia (10\%), normochromic 
normochromic (38\%), macrocytic anemia (4\%), dimorphic anemia (4\%) on the basis of peripheral smear.

Bone marrow studies were done in all cases of dimorphic and macrocytic picture.

It was noted that in the present study (48\%) of elderly patients showed iron deficiency anemia which was closely followed by anemia of chronic diseases (38\%) which consisted of chronic kidney disease, osteomyelitis, pneumonia, tuberculosis and carcinomas, no cause anemia $(6 \%)$, B 12 deficiency $(4 \%), B 12+$ Iron deficiency $(4 \%)$.

Bone marrow was done in 4 cases with peripheral smear features of microcytic and macrocytic anemia.

Iron deficiency anemia (47\%) was the predominant type of anemia seen in 30 elderly females followed by Anemia of chronic disease (43\%), No cause (7\%), B12 + Iron deficiency (3\%).

Iron deficiency anemia (50\%) was found to be the most common cause of anemia in 20 elderly males which was closely followed by anemia of chronic disease $(30 \%)$, B12 deficiency (10\%), No cause $(5 \%)$, B12 + Iron deficiency (5\%).

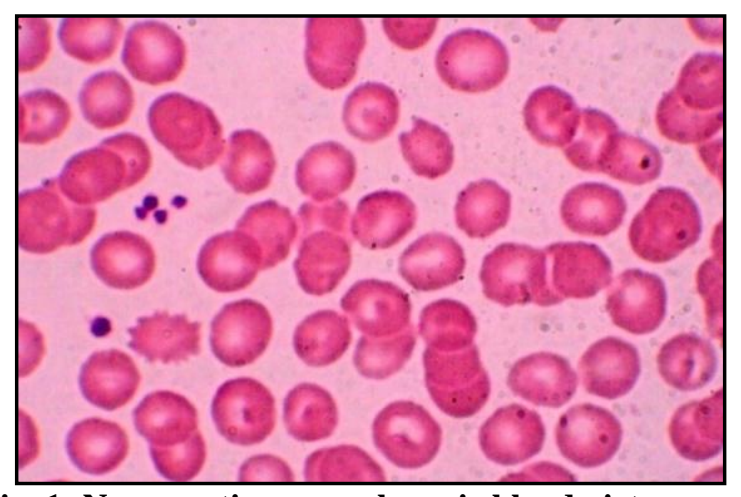

Fig. 1: Normocytic normochromic blood picture (Leishman's stain)

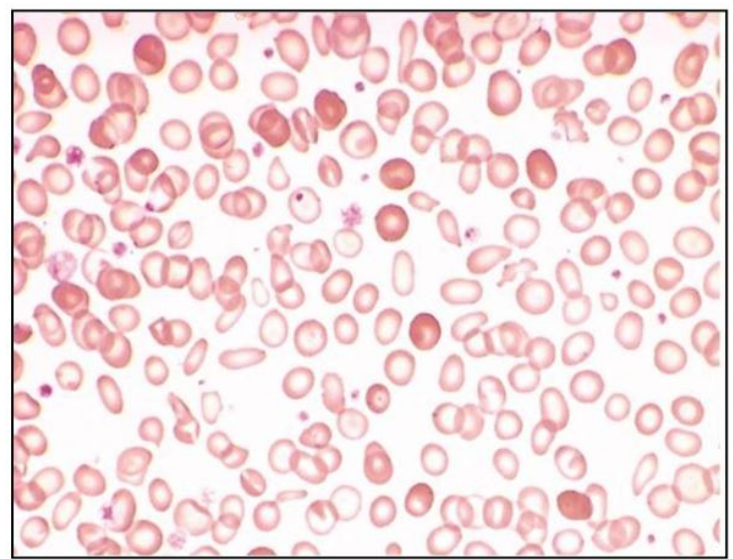

Fig. 2: Microcytic hypochromic blood picture (Leishman's stain)

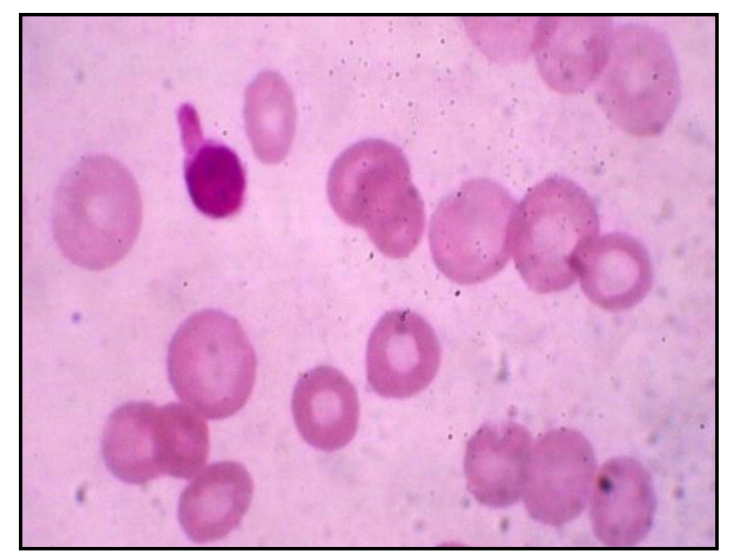

Fig. 3: Macrocytic blood picture (Leishman's stain)

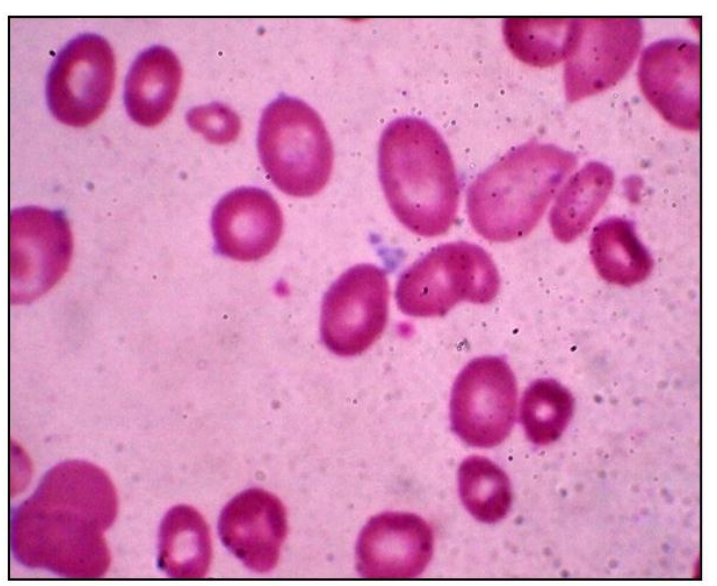

Fig. 4: Dimorphic blood picture (Leishman's stain)

\section{Discussion}

This study was undertaken to calculate the prevalence and to document the pattern of anemia in the three most common age groups affected by anemia that is in women of reproductive age group and elderly.

In our study, $6 \%$ of elderly patients had no obvious underlying cause. Artz et al ${ }^{14}$ showed that $14 \%$ to $50 \%$ of anemic elderly had no obvious underlying cause.

NHANES-III of $\mathrm{WHO}^{15}$ study revealed prevalence of anemia in $11 \%$ of men and $10.2 \%$ of women aged 65 years and older. However our"s was a hospital based study as compared to this study which was population based. In this hospital prevalence of anemia calculated was $71 \%$ in elderly males and $81 \%$ in females which is higher as compared to above study.

According to WHO in India $88 \%$ of pregnant and $74 \%$ of non pregnant women are affected. ${ }^{16}$ In our study it has been noted that anemia in pregnant women accounts for $72 \%$ and non pregnant women accounts for $70 \%$ which corroborates with WHO findings.

Divakar H, Nandakumar BS, Manyonda $\mathrm{IT}^{17}$ conducted a study on Iron deficiency anemia in pregnancy in $2006-2007$ and found an iron deficiency anemia prevalence of $69.4 \%$ in the rural and $61.4 \%$ in the urban setting. Our study shows a much more higher prevalence of iron deficiency anemia to be $88 \%$. 
In our study in elderly, microcytic hypochromic anemia was the most prevalent anemia accounting for $44 \%$ of all the cases closely followed by normocytic normochromic anemia accounting for $38 \%$.

Chernetsky et $\mathrm{al}^{18}$ has shown that most common anemia is anemia of chronic disease (65.6\%). Anemia primarily found due to Iron, VitB12 or folate deficiency was found to be only $4 \%$. Whereas in our study, a significant proportion of the cases accounted for this.

This difference may be accounted on basis of difference in the dietary intake and dietary patterns between the two populations.

In our study in elderly, $48 \%$ had IDA and $4 \%$ had B12 deficiency. Jack $M$ et al ${ }^{19}$ revealed that $16.6 \%$ of the patients had only iron deficiency, $6.4 \%$ had folate deficiency only and $5.9 \%$ of the patients had B12 deficiency. Hence our study corroborates with the findings of this study, iron deficiency being the most common of the nutrient deficiency anemia.

\section{Conclusion}

Confirming the type of anemia is critical to direct the investigation for profiling the etiology since it is well known that the treatment of anemia goes a long way in improving the overall outcome and quality of life.

Iron deficiency anemia is a serious problem worldwide and a major concern in developing countries. In our study we found to have higher prevalence of iron deficiency in all the study subjects. The main reason behind this nutritional anemia could be due to low socio economic status, increased demands, low dietary intake and excessive loss of blood, the possible causes of iron deficiency anemia in this region.

The implementation of national nutrition plans including the control of iron deficiency as one of the priorities and the participation of the public health and education sectors, food industries, the community and the media should contribute to the success of the interventions and to the control of iron deficiency. There is now need for the development of clear policy guidelines based on these simple and integrated interventions.

\section{References}

1. World Health Organization. The World Health Report 2002: Reducingrisks, promoting healthy life. Geneva;2002.

2. Macgregor M. Maternal anaemia as a factor in prematurity and perinatal mortality. Scottish Medical Journal, 1963; 8:134.

3. Scholl TO, Hediger ML. Anemia and iron-deficiency anemia: compilation of data on pregnancy outcome. American Journal of Clinical Nutrition, 1994;59:492S500 S.

4. Bothwell T, Charlton R, eds. Iron deficiency in women. Washington DC. Nutrition Foundation. 1981.

5. Guidelines for the treatment of malaria. Geneva, Roll Back Malaria Department, World Health Organization.
2006; WHO/HTM/MAL/2006.1108).

6. Crompton D.W.T, Montresor A, Nesheim M.C, Savioli L. Controlling disease due to helminth infections. Geneva, World Health Organization, 2003.

7. Mc Farlene DB, Pinkerton PH, Dagg JH and Goldberg A. Incidence of iron deficiency with and without anemia in general practice. Brit J Haemet. 1967;13:790.

8. Cook JD. Nutritional deficiency and anemia in Latin America. Acollaborative study. Blood. 1971;38: 5.

9. Celestin-Roux C, Hale WE, Perkins LL. "Anemia: an evaluation of age, sex, disease and medications in a geriatric population". J Geriatr Drug Ther. 1987;1:6386.

10. Fleming AF. Tropical obstetrics and gynaecology. 1. Anaemia in pregnancy in tropical Africa. Trans R Soc Trop Med Hyg. 1989;83:441-8.

11. Elis A, Ravid M, Manor Y, Bental T, Lishner M. "A clinical approach to idiopathic normocytic-normochromic anemia", J Am Geriatr Soc, 1996; 44:832-4.

12. Katai E, Kaplan B, Raick Y, Cohen Y, Neri A, Friedman J. Communityscreening to reveal iron deficiency in healthy menstruating women in Israel suburbs. Eur $J$ Obstet Gynecol Reprod Biol. 1996;67:21-5.

13. Iron deficiency anaemia: assessment, prevention, and control. A guidefor programme managers. Geneva, World Health Organization. 2001; (WHO/NHD/01.3).

14. Artz AS, Fergusson D, Gerald M. "Mechanisms of unexplained anemia in the nursing home". $J$ Am Geriatr Soc. 2004;52:423-7.

15. Nutritional anemias. "Report of a WHO scientific group". WHO technical support series 405 . World health organization, Geneva. 1968.

16. Jon C Aster. Robins and Cotron, Pathologic Basis of Disease, 7th ed. Pennsylvania, Elsevier Publishers. 2005:13, Red Blood Cell and Bleeding Disorders; 619660.

17. Divakar H, Nandakumar BS, Manyonda IT. Iron deficiency anemia in pregnancy: Is intravenous iron sucrose an alternative to the oral iron-folate supplementation program in India? Available at http://fkilp.iimb.ernet.in

18. Chernetsky A, Sofer O, Rafael C. "Prevalence and etiology of anemia in an institutionalized geriartic population". Harefuah. 2002;141:591-4,667.

19. Jack M. Guralnik et al in 2004 revealed that overall, $11.0 \%$ of men and $10.2 \%$ of women 65 years and older and living in the community are anaemic according to WHO criteria. 